\title{
Bureaux arabes
}

\section{Yacono}

\section{OpenEdition}

Journals

Édition électronique

URL : http://journals.openedition.org/encyclopedieberbere/1882

DOI : 10.4000/encyclopedieberbere.1882

ISSN : 2262-7197

\section{Éditeur}

Peeters Publishers

\section{Édition imprimée}

Date de publication : 1 septembre 1992

Pagination : 1657-1668

ISBN : 2-85744-581-4

ISSN : 1015-7344

\section{Référence électronique}

X. Yacono, «Bureaux arabes », Encyclopédie berbère [En ligne], 11 | 1992, document B115, mis en ligne le 01 avril 2013, consulté le 24 septembre 2020. URL : http://journals.openedition.org/ encyclopedieberbere/1882 ; DOI : https://doi.org/10.4000/encyclopedieberbere.1882

Ce document a été généré automatiquement le 24 septembre 2020

(c) Tous droits réservés 


\title{
Bureaux arabes
}

\author{
X. Yacono
}

1 Dans le cadre qui nous est proposé, le but de cette notice n'est pas d'étudier l'influence des Bureaux arabes sur la politique de la France en Algérie, mais seulement leur action sur le territoire algérien avec, dans la mesure du possible, car les études restent à faire, référence au monde berbère et plus spécialement à la Grande Kabylie.

\section{Origine et organisation}

\section{Une nouvelle administration}

2 Au lendemain de l'occupation d'Alger se posa un problème dont l'importance devait aller croissant avec la conquête, celui de l'administration des indigènes. On hésita longtemps entre le système turc consistant à confier cette tâche à un "agha des Arabes » et la création d'un organisme français de caractère original dont la première tentative, en 1832, prit le nom de « cabinet arabe ». Il n'y eut pas moins de huit essais différents et c'est le 16 août 1841 que fut prise l'option définitive avec la fondation par Bugeaud de la « Direction des affaires arabes » sous l'autorité du commandant Daumas. L'extension $\mathrm{du}$ territoire soumis à l'autorité française renforça la nouvelle administration qui affirma son importance, se diversifia et il fallut lui donner un statut: ce fut l'arrêté ministériel du $1^{\text {er }}$ février 1844 , considéré comme l'acte de naissance des Bureaux arabes, que compléta un code de mesures administratives et judiciaires applicables aux tribus. Cet arrêté fut seulement modifié et surtout précisé par la circulaire de Mac-Mahon du 21 mars 1867.

3 La hiérarchie des Bureaux arabes était en rapport direct avec celle du commandant de laquelle elle dépendait :

- les bureaux de deuxième classe assistaient les officiers supérieurs placés à la tête des cercles;

- les bureaux de première classe se trouvaient auprès des généraux commandant les subdivisions ;

- les uns et les autres dépendaient de la direction des affaires arabes de leur province 
laquelle était sous l'autorité du général commandant la Division ;

- au début, indépendamment de ses attributions, la direction divisionnaire d'Alger centralisait le travail des directions d'Oran et de Constantine, mais dès 1845 était nommé un directeur central des affaires arabes, sous les ordres immédiats du gouverneur général.

4 La circulaire du 21 mars 1867 mit à la tête des affaires arabes un bureau politique et officialisa la création de bureaux annexes de cercle. A la fin du Second Empire, il existait en Algérie 41 bureaux arabes de première et deuxième classes et cinq annexes.

Parmi ces bureaux arabes nombre d'entre eux étaient en pays berbère sans que les limites des circonscriptions fussent nécessairement ethniques ou linguistiques. Mais l'étude de cette institution en Grande Kabylie et dans les Aurès, pour se limiter à ces deux régions essentielles, impliquerait, pour le moins, le dépouillement des archives concernant les bureaux suivants :

- dans la division d'Alger : Aumale (subdivision en 1849) pour la partie septentrionale ; Beni-Mansour (au pied du Lalla Khedidja) ; Dellys (subdivision en 1858) ; Fort-Napoléon qui deviendra Fort-National ; Tizi-Ouzou ; Dra-el-Mizan.

- dans la division de Constantine : Bougie, Akbou, Aïn Beïda ; Batna (subdivision en 1847); Khenchela.

\section{Fonctions et pouvoirs}

6 Le but poursuivi est clairement indiqué par Daumas : «Cette institution, écrit-il, a pour objet d'assurer la pacification durable des tribus par une administration juste et régulière, comme de préparer les voies à notre colonisation, à notre commerce, par le maintien de la sécurité publique, la protection de tous les intérêts légitimes et l'augmentation du bien-être chez les indigènes. »Cela supposait non seulement la participation active à la conquête, mais une connaissance approfondie de la société indigène, de ses intérêts, des forces qui agissent sur elle afin de définir, dans tous les domaines, une action susceptible de la transformer et de l'amener « à accepter avec le moins de répugnance possible et notre domination et les éléments du gouvernement qui doivent l'affermir ». Isolés au milieu de tribus le plus souvent hostiles, les officiers des affaires arabes devaient se montrer à la fois hommes de guerre, diplomates, administrateurs, directeurs de travaux publics, inspecteurs des contributions, conseillers agricoles, juges, directeurs de l'enseignement... Pour reprendre un mot de Lyautey, seul pouvait réussir « un civil intelligent dans la peau d'un militaire ».

7 Encore fallait-il qu'il disposât des pouvoirs nécessaires. Or toutes les instructions à partir de l'arrêté fondamental de 1844 précisent bien que les Bureaux arabes sont des agents d'exécution, qu'ils ne constituent pas un corps spécial mais un simple "trait d'union", un "rouage" et que toute l'autorité appartient aux commandants des circonscriptions militaires auxquels ils doivent toujours rendre compte de leur action et demander des directives. Dès 1849 cependant, Walsin-Esterhazy, à la direction des affaires arabes à Oran, écrivait : c'est « par une fiction nécessaire peut-être à l'unité de commandement» que "les bureaux arabes sont censés n'être que des instruments entre les mains des commandants supérieurs des subdivisions et des cercles souvent étrangers aux choses arabes ». En réalité, spécialistes en contact direct avec les indigènes dont ils parlaient souvent la langue (l'arabe ou le kabyle, parfois les deux), ayant à résoudre les mêmes problèmes, se heurtant aux mêmes difficultés, mal 
considérés au début par les autres officiers les accusant de ne plus être de véritables militaires, ils en arrivèrent à constituer une "puissante confrérie " qui devint comme le symbole $\mathrm{du}$ "régime $\mathrm{du}$ sabre ». Ce pouvoir que les textes leur déniaient, ils l'acceptèrent et on pourrait en multiplier les preuves. Rapportons, par exemple, ce qu'écrit Rinn, ancien chef de bureau arabe et historien: «Les indigènes habitués à n'avoir qu'un seul chef, le bureau arabe... le tenaient en très haute considération et ils n'avaient qu'un souci relatif des autorités dont dépendait le bureau. Ils savaient bien que le commandant supérieur était le chef de leur circonscription et qu'au-dessus, il y avait encore des généraux : un petit (à la subdivision), un grand à la division et, plus haut encore, le maréchal, c'est-à-dire le gouverneur; mais ils voyaient rarement ces hauts personnages et ils n'avaient guère affaire à eux, si ce n'est pour les actes de courtoisie, et le bureau arabe était tout. » Quant à Pein, qui servit au bureau arabe de Bou-Saada, il s'écrit: «Eh bien, moi aussi j'étais roi, je jouissais d'une liberté sans bornes; les grandes tribus de cavaliers du Hodna, ces tribus nomades des Oulad Naïl, ne reconnaissaient de chef que moi, n'obéissaient qu'à moi. »

\section{Les moyens d'action}

8 Dans ces conditions, il est évident que la qualité du personnel était l'élément fondamental. Or, contrairement à ce qu'écrivirent leurs adversaires, les officiers qui optèrent pour les affaires arabes, surtout ceux de la première génération, ne furent pas des médiocres mais l'élite de l'armée et cela se comprend: des fonctions aussi complexes devaient tenter surtout des hommes de caractère, aux aptitudes diverses, poussés sans doute par l'amour du pouvoir mais aussi, chez beaucoup, par un véritable idéal et le désir de servir ce qu'ils pensaient être une grande cause. Issus dans une forte proportion des Écoles d'officiers (60\% dans l'Algérois de 1844 à 1856) et notamment de Polytechnique, lieutenants ou le plus souvent capitaines, c'étaient des hommes jeunes, ambitieux, qui avaient parfois subi l'influence des grands mouvements d'idées de l'époque : le catholicisme libéral et social, les théories socialistes de Fourier et de SaintSimon notamment.

9 Citons quelques noms parmi ceux qui commandèrent en Grande Kabylie. Jean-Charles Devaux sert à Beni-Mansour et à Dra-el-Mizan et en 1859, il publie Les Kebailes du Djerdjura, important ouvrage de 468 pages où il traite des mœurs des habitants et présente leur organisation en clans et confédérations ; il donne aussi un recueil sur les divers kanouns des villages de la Grande Kabylie. Aucapitaine qui devait passer par FortNapoléon, Dellys et Beni-Mansour, publie plusieurs études sur la Kabylie et notamment, en 1864, une brochure de 180 pages intitulée Étude sur le passé et l'avenir des Kabyles et la colonisation de l'Algérie dans laquelle il conseille de favoriser l'évolution des Kabyles et d'en faire les éducateurs des Arabes. Gaston de Sonis commanda le bureau arabe de Fort-Napoléon de 1859 à 1861 avant de s'illustrer dans le Sud et pendant la guerre contre la Prusse comme général commandant le $17^{\mathrm{e}}$ corps d'armée. Le polytechnicien Hanoteau, qui commanda à Dra-el-Mizan, Fort-Napoléon et Dellys, publie en 1867 un important recueil (475 p.) de Poésies populaires de la Kabylie du Djurjura, texte et traduction; puis en 1872, avec la collaboration de Letourneux, conseiller à la Cour d'appel d'Alger, une œuvre capitale en trois forts volumes, La Kabylie et les coutumes kabyles. Au total, un personnel peu nombreux et, à la fin du Second Empire, moins de 200 officiers servaient dans les Bureaux arabes, dont un tiers peut-être en pays berbère, et administraient plus de deux millions d'Algériens. 
10 Avec quels moyens? On a pu estimer à seulement $1 \%$ la part du budget algérien consacré aux Bureaux arabes, ce qui en faisait une administration particulièrement économe. Il faut cependant préciser que le service des affaires arabes disposait de ressources, qui, pour ne pas être inscrites au budget, n'en étaient pas moins importantes. Il y avait tout d'abord les centimes additionnels à l'impôt arabe, et sous le nom de " dépenses communales ", un certain nombre de dépenses auxquelles devaient pourvoir les tribus comme, par exemple, la nourriture des chevaux et des hommes du makhzen en tournée. Il existait aussi la possibilité de faire appel à des corvées et réquisitions, donnant lieu en principe à indemnisation mais pouvant aboutir à des abus. Le chef de bureau arabe exigeait parfois, pour des agents indigènes ou pour lui-même, une corvée spéciale, la touïza, visant à mettre en culture certaines terres et le capitaine Beauprêtre, chef de l'annexe de Beni-Mansour, fut accusé d'avoir fait cultiver à son profit une superficie assez vaste (50 hectares ?) pour lui avoir rapporté un revenu de 12 $000 \mathrm{~F}$ par an. Cependant le contrôle fut de plus en plus efficace et il est certain que si les ressources marginales et les fonds secrets existèrent toujours, il eût fallu des possibilités autrement considérables pour assurer l'action économique et sociale à laquelle songèrent les Bureaux arabes.

11 Dans le domaine politique par contre, ils étaient mieux armés grâce à l'appui de la haute administration militaire et à l'institution du makhzen, empruntée aux Turcs, et qu'Azéma de Montgravier définit comme un « corps de cavalerie indigène, auxiliaire, irrégulière et privilégiée ». Pour Lapasset, les hommes du makhzen sont « les yeux, les oreilles, les bras, les jambes » du bureau arabe et pour Richard, chef du bureau arabe d'Orléansville, c'est «l'instrument de l'ordre ». Ce makhzen ne fut cependant jamais très nombreux et chaque chef de bureau arabe n'eut à sa disposition que quelques dizaines d'hommes mais bien montés, bien armés et connaissant à merveille le pays, ils constituaient le noyau autour duquel, en cas de besoin, se groupaient d'autres éléments de tribus fidèles formant ce qu'on pourrait appeler «le parti français ", résultat de l'action politique et militaire des Bureaux arabes.

\section{L'action politique et militaire}

\section{La création du parti français}

12 Puisque «dans ce pays l'aristocratie est tout et le peuple n'est rien » (Montgravier), c'est évidemment sur l'aristocratie qu'il faut s'appuyer pour agir et créer un parti qui non seulement acceptera la domination française mais en facilitera l'établissement.

13 Ces chefs dont le recrutement dépend essentiellement des Bureaux arabes seront choisis, à défaut des grandes familles de marabouts ou de djouad, parmi les notables jouissant d'un certain prestige. On fera donc appel à ceux qui traditionnellement exercent l'autorité, surtout lorsqu'il s'agit de khalifas, bachagas, aghas. Quelques familles, jugées irréconciliables, sont, dans l'immédiat tout au moins, écartées du commandement, comme les Berkani du cercle de Cherchel. Le cas est assez rare et souvent on réserve aux ralliés un sort privilégié : en 1847, par exemple, Omar Ben Salem, qui vient de faire acte d'allégeance, obtient le titre de bachaga de l'Oued Sahel au détriment d'autres chefs fidèles depuis plus longtemps et le cas n'est pas exceptionnel. Il s'agit chaque fois de trouver l'homme qui, par son influence, est le plus susceptible de ramener l'ordre. On risque évidemment de s'attirer de nouveaux 
ennemis. D'où un jeu subtil de bascule à mener entre les familles, et parfois à l'intérieur d'une même famille, pour ménager l'amour-propre et les ambitions de chacun.

La confiance dans ces chefs traditionnels demeurait cependant médiocre et beaucoup pensaient, comme le capitaine Richard, qu'ils « sont obligés, dans leur position délicate, d'avoir, comme Janus, deux faces opposées. Pour nous qui les employons et qui payons leurs services au poids de l'or, ils ont la face du dévouement et de l'intelligence avancée qui comprend nos idées et veut nous aider à les appliquer; pour le peuple qu'ils administrent, ils ont celle de la pureté musulmane qui souffre à notre contact et ne supporte notre domination que comme une calamité passagère à laquelle il faut se soumettre jusqu'à ce que Dieu l'ait fait disparaître ».

Dès lors l'autorité française s'efforce d'une part d'affaiblir progressivement les élites traditionnelles et, d'autre part, d'en faire surgir d'autres. La charge de Khalifa n'est plus accordée. On hésite à réduire l'autorité des chefs qui ont rendu de réels services mais lorsqu'ils disparaissent, leurs héritiers n'obtiennent pas les mêmes pouvoirs. On donne une importance accrue au recrutement des caïds issus du makhzen, simples chaouchs parfois ou cavaliers du bureau arabe et, plus encore, spahis. Ce sont là gens de petite extraction qui n'avaient pas le prestige des notables traditionnels mais étaient beaucoup plus dociles dans les mains des Bureaux arabes. Ainsi on évoluait vers une administration qui prenait de plus en plus les caractères d'une administration directe.

Il faudrait mettre à part la politique menée auprès des chefs religieux et des confréries dont la force se révéla surtout avec l'insurrection de 1845. Les Bureaux arabes n'adoptent pas une politique hostile à l'Islam et, en Kabylie notamment, l'islamisation était considérée comme une étape dans la voie de la civilisation, une moralisation relative des indigènes et, par suite, un moyen d'améliorer l'action du gouvernement. Par contre l'évangélisation apparaissait comme un danger et le bureau arabe s'en prend à l'action des missionnaires au point que Mgr Lavigerie rend Hanoteau responsable de l'échec de sa politique évangélique. Mais cette attitude ne signifie pas que les Bureaux arabes étaient favorables aux confréries, considérées avec méfiance parce que secrètes et entretenant des relations à l'extérieur (Maroc, Turquie). C'étaient essentiellement les Taïbia, les Derkaoua, les Kadirya, les Tidjanya et les Rahmanya, ces derniers dominant l'est de l'Algérie et notamment dans la Grande Kabylie. Si les Tidjanya avaient plutôt soutenu les Français contre Abd el-Kader, les autres confréries paraissaient beaucoup moins favorables et les Bureaux arabes s'efforçaient de les contrôler, en apparence étroitement (dans leurs rapports ils fournissaient même le nombre des affiliés), en réalité difficilement.

Quel fut le résultat de cette action? Ce ne fut certes pas un succès comme devait le montrer la grande révolte de 1871, mais peut-on parler d'échec général? Il y eut rapidement à Fort-Napoléon un parti français et le makhzen du caïd El Hadj Ahmed Iattaren ainsi que les goums de Belkassem ou Kassi rendirent de grands services au bureau arabe en arrêtant de nombreux voleurs et meurtriers. A partir de 1874, des gardes champêtres indigènes furent chargés spécialement du contrôle des forêts et de la police des montagnes du cercle de Fort-National. Pendant la grande révolte même, l'accord ne fut pas général contre les Français et une enquête sur les chefs indigènes dénombre 13 chefs demeurés favorables à la France contre 16 ayant pris le parti de l'insurrection. 


\section{La diplomatie et la conquête}

18 Participer à la conquête et à la pacification du pays, puis maintenir l'ordre, tel était évidemment le but premier de l'action des Bureaux arabes. En pays berbère comme ailleurs, ils jouèrent souvent un rôle de premier plan.

Dans la conquête de la Kabylie, ils constituent l'avant-garde des colonnes françaises. La stratégie générale est fixée par Daumas en 1847 : «Maintien des formes républicaines de la tribu (Kabyle), délégation de l'exercice du pouvoir à des marabouts, emploi judicieux des çoffs et des grandes familles qui les dominent pour appuyer notre centralisation sur celle même que les tribus acceptent, et investir précisément les hommes dont l'influence personnelle est déjà reconnue; respect aux lois antiques du pays, à ces kanouns traditionnels qui d'ailleurs ne froissent en rien les principes du droit public. Ces bases posées, notre édifice n'aura plus de secousses à craindre que sur le terrain des impôts. »

Mais auparavant, il fallait pénétrer dans le pays qui avait échappé à la domination turque, et tout d'abord l'investir. D'où toute une politique d'encerclement se manifestant par la création de smalas sur les axes de communication et par des manœuvres d'approche menées par les Bureaux arabes auprès de certains chefs indigènes comme Ben Zamoun et Ben Zitouni en pays Flissa, Bel Kassem ou Kassi dans la vallée du Sebaou et surtout Si Djoudi (marabout de la zaouïa d'Igoulmimène dans les Ouadia) et Ben Ali Chérif (marabout de Chellata), ce dernier influent sur les tribus de la moyenne vallée de l'oued Sahel. Les résultats de cette diplomatie furent médiocres et pour établir l'autorité française sur les tribus limitrophes des Zouaoua, au cœur du pays insoumis, il fallut intervenir par la force avec les goums, en particulier celui du lieutenant Beauprêtre qui appartenait alors au Bureau arabe d'Aumale. En 1850, la Kabylie était déjà largement investie au nord, à l'ouest et au sud.

21 De 1851 à 1856, l'action militaire proprement dite marque le pas, mais les Bureaux arabes s'efforcent toujours de faire pénétrer l'influence française en Kabylie non sans difficultés car c'est l'époque de l'insurrection de Bou Baghla (1851-1854) qui permet un moment aux tribus hostiles de desserrer l'étau qui se refermait sur elles. Le commandant français y répond en essayant de fractionner les tribus, en utilisant les oppositions entre çoffs et entre individus puis en réorganisant le makhzen du Haut Sebaou. A cause de circonstances extérieures (guerre de Crimée), c'est une politique d'attente dans laquelle les Bureaux arabes jouent un rôle important. Ils ne peuvent empêcher une nouvelle insurrection d'éclater en août 1856, mais à ce moment le gouverneur Randon est prêt à intervenir. S'incorporant aux différentes colonnes, les officiers des bureaux arabes qui avaient mené l'action diplomatique (commandant Péchot, capitaines Colonieu, Bonnefont-Pédufau, Gandil, lieutenants de Saint-Joseph, Senaux, Jobst, Cassignol) feront bénéficier le commandement de leur connaissance du pays et des hommes. Ce sera la campagne de 1857 que nous n'avons pas à raconter.

Suivent alors une dizaine d'années au cours desquelles les Bureaux arabes poursuivent une politique économique et sociale que nous définirons plus loin. La soumission kabyle n'est qu'apparente et comme le note un rapport de quinzaine de Fort-Napoléon en 1859 : « une foule de nouvelles circule ayant toutes plus ou moins trait à la question de notre domination en Algérie. » Il y eut en Grande Kabylie des complots ou des velléités de révolte chaque fois que des actes de rébellion se manifestaient quelque part en Algérie ou même en Tunisie (la révolte de 1864), notamment lors des mouvements en 
Kabylie orientale en 1858-1860 et en 1864-1865. Par contre l'annonce du retour d'Abd el-Kader accompagnant le réembarquement des Français fut mal accueillie: "La majorité de la population n'admet qu'avec la plus grande répugnance la possibilité de la réalisation de ces bruits. Pour eux, Abd el-Kader n'est qu'un Arabe et leur fierté se révolte à la pensée qu'il pourrait être appelé à les commander " (rapport mensuel de Fort-Napoléon d'août 1865). Seules les années économiquement difficiles de 1866 à 1868 sont politiquement calmes mais, dès 1868, les Bureaux arabes traduisent certaines inquiétudes que justifieront un début de révolte en août 1870 dans la région de Bougie et surtout la grande insurrection de 1871.

A propos de celle-ci, une question se pose quant à ses origines : quel fut le rôle des Bureaux arabes dans le déclenchement de la révolte? Depuis toujours les civils accusaient les militaires, et plus particulièrement les Bureaux arabes, de préparer sciemment, de temps à autre, un mouvement insurrectionnel plus ou moins important pour justifier le maintien du "régime du sabre » et procurer l'occasion aux officiers d'obtenir de l'avancement. Cette attitude des civils peut s'expliquer par leur hostilité à l'égard des Bureaux arabes qui s'étaient fait les défenseurs du patrimoine foncier indigène en combattant la politique du cantonnement. Dans leur ensemble les historiens ont rejeté ces accusations. Or, il se trouve que l'un d'entre eux, et non des moindres, Charles-André Julien, fait une exception pour l'insurrection de 1871. Il note, en effet, que loin d'essayer de diviser pour régner, l'armée, et en l'occurrence les bureaux arabes, s'est appliquée à mettre fin aux luttes entre les çoffs puis aux rivalités maraboutiques dans l'espoir de provoquer un mouvement limité qui favoriserait le maintien du régime militaire. Cela s'accorde mal avec le fait que les moyens de répression manquaient alors en Algérie et avec l'inquiétude que traduisent les rapports des responsables de la politique indigène, soulignant depuis longtemps le danger que représenterait pour la Kabylie une insurrection dans la province de Constantine. Mais il est certain qu'après l'écrasement de l'insurrection kabyle, le maintien des Bureaux arabes n'apparaissait plus comme une nécessité première.

\section{L'action économique et sociale}

Elle fut inspirée par des considérations humanitaires et politiques. C'est le responsable de la première "Direction des affaires arabes ", le capitaine Pellissier de Reynaud, qui affirme: "Il n'y a de conquêtes légitimes et durables que là où le peuple vainqueur élève à lui le peuple vaincu de manière à ce que l'avenir amène une fusion complète. » Mais cela n'exclut pas les visées politiques traduites dans de nombreux textes et exprimées en 1852 par Randon conseillant d'améliorer le bien-être de la population laborieuse car : «C'est en favorisant aussi largement que possible le développement de ce bien-être que je chercherai à modifier à notre avantage l'opinion politique des tribus.»

\section{Les essais d'amélioration économique}

Les Bureaux arabes entreprirent de développer la production et les échanges en introduisant les méthodes de l'économie française. Ils crurent possible de faire rapidement du paysan indigène un paysan adoptant un mode de vie importé. 


\section{a. La production} étaient insuffisantes et où il fallait mêler le bechna (sorgho) à l'orge et aux glands doux, il n'y avait parfois pas assez de grain pour ensemencer : d'où la constitution obligatoire de réserves. Surtout les arbres fruitiers (pommiers, poiriers, abricotiers, pêchers et d'abord figuiers et oliviers) constituaient une richesse essentielle et le bureau arabe de Fort-Napoléon fit appel à un jardinier français pour apprendre notamment aux indigènes à greffer les merisiers et les prunelliers. Il accorda des primes à certains planteurs et aurait voulu modifier le contrat coutumier qui ne leur accordait que le tiers ou le quart des récoltes contre les deux tiers ou les trois quarts au propriétaire. Les plantations de frênes furent encouragées le long des chemins et pour limiter les champs.

Les Bureaux arabes voulurent aussi réformer les conditions d'exploitation en vue d'augmenter les rendements. On vit les officiers recommander l'emploi de la charrue française (perfectionnée par Mathieu de Dombasle), de la herse, de la faux, se heurtant à l'obstacle du relief montagneux et à la nature des ressources car, comme l'écrit l'un d'eux: «l'usage de la faux n'a pas grande chance de prévaloir dans le pays où les prairies sont rares."

On songea aussi à introduire des cultures nouvelles et, en premier lieu, celle de la pomme de terre destinée, en Kabylie, à remplacer avantageusement le chêne à glands doux. Les résultats furent d'abord encourageants mais les indigènes préférèrent commercialiser la récolte auprès des Européens plutôt que la consommer. La vigne eut plus de succès et les Kabyles vendirent le raisin aux habitants de Fort-Napoléon à des prix rémunérateurs : 25 centimes le kilo en 1860, 30 centimes en 1862. La pépinière créée à Fort-Napoléon fournit de nombreux pieds de châtaigniers et le jardin militaire ne suffit pas pour répondre à la demande en pommiers, amandiers, orangers. On essaya aussi les plantations d'eucalyptus et de tabac ainsi que les ensemencements de luzerne, mais avec peu de résultats.

L'industrie artisanale avait toujours joué un rôle notable et elle fut rapidement menacée par la concurrence européenne. Les Bureaux arabes défendirent l'activité des ouvriers travaillant le bois (fabrication de couverts et de plats) et essayèrent de former des briquetiers et des maçons. Surtout les Zouaoua, et en particulier les Benni Yenni, développèrent la production des bijoux* filigranes, enrichis d'émaux ou de cabochons de corail qu'on put admirer à l'exposition permanente des produits algériens à Paris et aux expositions universelles de Paris en 1867 et de Vienne en 1874.

La modernisation intéressa essentiellement l'industrie de l'huile. Très tôt, aux frontières de la Kabylie, à Dellys, à Aumale, des négociants français effectuèrent des achats d'huile et dès 1851 des contrats passés devant le cadi ou le bureau arabe fixaient la quantité et le prix de l'huile à livrer. Après la conquête, des fabricants d'huile s'établirent au cœur du pays, à Fort-Napoléon, et les Kabyles trouvèrent avantage à leur apporter les olives : en 1870, il y avait quatre « usines à huile » dans le cercle.

\section{b. Les échanges}

31 Ceux-ci peuvent être effectués sur les marchés locaux et, sous le Second Empire, le cercle de Fort-Napoléon, par exemple, en compte 16 dont 2 créés par le bureau arabe. La police y est assurée par l'amin el oumena sous le contrôle de l'autorité militaire.

Encyclopédie berbère, 11 | 1992 
Mais ces marchés sont autant sinon plus des lieux de réunion que de commerce et, en pays kabyle, les véritables échanges se font avec l'extérieur.

Dès 1858, le bureau arabe entreprend la construction de la première route, celle du col de Tirourda. Les travaux se poursuivent avec l'aide des Kabyles qui, selon les officiers, firent preuve "de beaucoup d'entrain et de bonne humeur", surtout pour la construction des ponts. L'inauguration de ces derniers s'accompagnait de fêtes réunissant des milliers de Berbères : 6000 pour le pont sur l'oued Djemâa destiné à joindre les Beni Attaf et les Beni Menguellet.

Bien avant l'arrivée des Français, les Kabyles exportaient leurs productions et leurs bras. Le mouvement ne fit que s'amplifier, allant des frontières de la Tunisie à celles du Maroc avec comme pôles d'attraction essentiels Alger et, plus encore, Constantine. Les exportations portent sur l'huile, les figues, la cire, les produits de l'artisanat local mais aussi des bœufs et des chevaux que les Kabyles allaient chercher au loin pour les engraisser avant de les revendre. Chaque année, après l'Aïd-es-Seghir partent les colporteurs, les "attars ", munis d'un passeport du bureau arabe et, en août 1861, à Fort-Napoléon, on délivre 100 passeports par jour, le total s'élevant d'environ 2600 en 1857 à 15000 en 1874 (au moment où les Kabyles sont soucieux de payer les soultes du séquestre) avec une interruption pendant les années de famine de 1867-1868. Le moyen de transport est le mulet et les seuls Beni Raten en utilisent 300. En 1866, on voit même trois Kabyles partir pour Marseille «comme pouvant leur offrir de bons débouchés pour leurs produits ». Le bureau arabe de Fort-Napoléon favorise aussi le départ des journaliers agricoles qui allaient en grand nombre dans la Mitidja travailler pour 3 francs par jour (le prix de l'orge à l'époque varie entre 10 et $20 \mathrm{~F}$ l'hectolitre).

Lorsque les échanges sont fructueux, les Bureaux arabes encouragent les travaux publics comme l'édification de fontaines ou la construction de maisons à l'européenne pour lesquelles on fit marcher à plein rendement certaines carrières. Le résultat fut parfois assez déconcertant : alors qu'en pays arabe on s'efforçait de grouper en villages le nouvel habitat, en Kabylie, on vit, contrairement à la coutume, s'élever des maisons isolées!

\section{Les transformations sociales}

Nous limiterons notre étude à l'enseignement et à la santé.

\section{a. L'enseignement}

S'il était moins développé qu'en pays arabe, l'enseignement musulman existait cependant en pays berbère et les chefs de bureau arabe signalent l'existence de zaouïas notables, par exemple chez les Beni Fraoussen (la zaouïa de Djema Saharidj), les Beni Raten, les Beni Aïssa, les Beni Mansour. Il est difficile de préciser l'importance de l'enseignement élémentaire et le niveau de l'instruction, mais il est certain que peu connaissaient bien la langue arabe (le gouvernement général s'étonnera du faible nombre d'abonnés au Mobacher) et ceux qui la connaissaient répugnaient souvent à la parler. En tous cas les Bureaux arabes se montraient indifférents et parfois farouchement hostiles à ces écoles traditionnelles dans lesquelles ils voyaient "des foyers de fanatisme... le lieu de réunion de tous les ennemis les plus acharnés de notre domination ». 
37 La solution parut être la création d'écoles arabes-françaises où, en principe, on enseignait l'arabe le matin et le français le soir. Comme exemple, on peut citer celle de Fort-Napoléon ouverte en 1860 dans un local donné par un colon et qui groupa cette année-là 12 indigènes et 7 Européens. Dirigée par un caporal, parfois obligé de s'absenter, manquant de matériel, son succès fut limité. Quant à l'ouvroir pour les filles auquel pensait le bureau arabe, il demeura à l'état de projet.

Les Pères Blancs avaient ouvert plusieurs écoles en pays berbère et, en 1872, on en comptait trois dans la région de Fort-National dont celle de Djema-Saharidj. Soutenue plus ou moins par l'autorité militaire supérieure qui y voyait une «action salutaire... dans la conquête morale du peuple », cet enseignement ecclésiastique ne plaisait pas toujours aux Bureaux arabes qui, hostiles à tout ce qui semblait être une évangélisation, craignaient de soulever l'animosité de la population.

39 La grande création fut l'école des Arts et Métiers de Fort-Napoléon. C'est le commandant Hanoteau qui, dès 1860, avait eu l'idée de fonder une " école d'artisanat " où l'on apprendrait à travailler le fer et le bois et qui fonctionnerait sous la forme mutualiste: elle fabriquerait des objets utiles et les bénéfices que procurerait leur vente seraient répartis entre les élèves sous la forme d'une solde. L'école fut construite à 800 mètres du fort et elle s'ouvrit en 1866. Elle devait recevoir des élèves de 15 à 17 ans, originaires de la division d'Alger et qui tous étaient boursiers. Il en vint effectivement d'Alger, de Médéa, de Miliana, mais la majorité était de Kabylie. L'effectif maximum fut atteint en mars 1871 avec 94 élèves. Fermée en juillet, l'école, selon les rapports, fut détruite par ses propres élèves pendant la grande insurrection kabyle.

\section{b. La santé}

40 Contrairement à une idée qui eut longtemps cours, les montagnes n'étaient pas à l'abri des épidémies et en 1849 les Kabyles, notamment les Zouaoua, souffrirent sérieusement du choléra et, en 1854-1855, au choléra s'ajouta la variole qui attaque surtout les tribus des environs de Dellys (Flisse El Bahr, Beni Ouaguenoun, Beni Djennad, Amaraoua, Beni Slyem).

41 Dans le service de santé, les Bureaux arabes virent le moyen d'exercer à la fois une action humanitaire et politique car, comme l'écrit Lapasset en 1847 : le médecin dans les tribus « serait un puissant élément de civilisation; un lien de reconnaissance entre le vaincu et le vainqueur ; un moyen de moraliser la race arabe, de diminuer chez elle cet aveugle principe de la fatalité. Bien des renseignements que ne peuvent savoir les chefs de Bureaux arabes seraient recueillis par les docteurs, bien des nouvelles apprises par eux. »

C'était compter sans les réalités du pays et de l'institution. Créé par un arrêté du gouverneur général du 30 juin 1847, le service de santé gratuit auprès de chaque Bureau arabe voyait ses postes pourvus en médecins dès le début de 1848. Beaucoup de ceux-ci firent preuve d'un remarquable dévouement mais les ressources dont ils disposaient étaient ridiculement faibles, les moyens manquaient au point que des médecins durent acheter un cheval de leurs propres deniers pour effectuer des tournées dans les tribus.

Et l'attitude des populations indigènes ne fut pas celle qu'on attendait. Si les malades, essentiellement des hommes, acceptaient les soins courants surtout lorsqu'ils s'avéraient rapidement efficaces, ils répugnaient à entrer dans les hôpitaux français. La 
vaccination contre la variole souleva une opposition qui alla jusqu'à la révolte dans les subdivisions de Miliana et d'Aumale ainsi que dans le cercle de Philippeville. Peut-être les choses se seraient-elles passées sans incident, si, comme l'écrivit le prince Napoléon en juillet 1858, «l'autorité militaire avait pris soin de réunir préalablement les chefs arabes, de leur faire comprendre le but philanthropique que nous poursuivons, enfin, si elle n'avait agi qu'après avoir reçu d'eux l'assurance que l'opinion était préparée à accueillir favorablement nos médecins ». Quoi qu'il en soit il faudra de nombreuses années avant de faire admettre cette arme moderne et efficace.

Il est certain que ni économiquement ni socialement les Bureaux arabes ne sont parvenus à provoquer une évolution sérieuse de la population indigène et en particulier de la population berbère. Leurs ennemis expliqueront cet échec par l'attitude qu'ils avaient adoptée à l'égard de la colonisation.

\section{Les bureaux arabes et la colonisation}

45 A l'époque où l'action des Bureaux arabes se manifeste en Algérie, le pays berbère est encore relativement peu atteint par la colonisation. Il parait cependant utile d'indiquer rapidement quels furent les caractères essentiels des rapports entre colons et officiers des Bureaux arabes.

Considérant la colonisation comme un moyen d'affermir l'occupation, les Bureaux arabes préconisent tout d'abord son extension, mais en même temps ils estiment indispensable la collaboration entre les deux éléments de la population. Lorsqu'ils penseront que celle-ci a échoué, ils changeront d'avis et d'attitude.

\section{Les alliés de la colonisation}

$47 \mathrm{Au}$ début donc, les officiers se dépensent pour inventorier les terres susceptibles de servir à la colonisation. Ils défendent même le principe du cantonnement car : « Il y a place pour tous sous le soleil d'Afrique. Les indigènes ont trop de terre, ils se resserreront; ils occuperont les cases noires de l'échiquier, tandis que les Européens occuperont les blanches; les deux colonisations s'avanceront de front vers l'intérieur du pays " (Lapasset en 1858: dans un autre texte, il place les Européens sur les cases noires et les indigènes sur les cases blanches). Aussi les Bureaux arabes apporteront-ils leur aide à la fondation des colonies agricoles de 1848 dont aucune toutefois ne devait être établie dans le véritable pays berbère.

Mais dès le début du Second Empire, les chefs des Bureaux arabes font preuve de prudence et soulignent les dangers d'un «resserrement excessif ». En Kabylie, ils attirent l'attention sur l'importance des biens melk et l'absence de domaine rural appartenant à l'État à l'exception de certains massifs boisés. Ils en arrivent à éveiller la méfiance du gouverneur général Randon qui, dans une lettre au général Yusuf, commandant la division d'Alger, exprime ses soupçons en janvier 1856: «J'ai eu l'occasion, écrit-il, de remarquer que les Bureaux arabes dépassaient parfois la mesure de la protection à laquelle peuvent légitimement prétendre les indigènes... Il ne faut pas que cette protection, portée jusqu'à l'abus, crée des entraves, que la population européenne recule devant la population arabe; qu'on marchande parcimonieusement le terrain à la première et que l'on prodigue l'espace à la seconde, sous le prétexte des 
habitudes agricoles. Quelques-uns des rapports que vous m'avez transmis indiquent cette tendance... »

\section{Les adversaires de la colonisation}

49 C'est que dans deux domaines au moins, les officiers des Bureaux arabes, se plaçant au point de vue des indigènes, ont tiré des faits, des conclusions qui n'étaient pas favorables à l'action colonisatrice.

50 Si le pays berbère échappa dans son ensemble au cantonnement, cette opération foncière y eut cependant des répercussions indirectes. En effet, le cantonnement qui aurait dû asseoir la propriété indigène par la distribution de titres réguliers aboutit, au contraire, à la désagrégation foncière, les nouveaux détenteurs de titres ayant désormais toutes facilités de s'en dessaisir par la vente. Les officiers des Bureaux arabes dénoncèrent ce danger et combattirent cette politique trop favorable à la colonisation. Aussi lorsque fut promulgué le sénatus-consulte du 22 avril 1863, en Kabylie, comme ailleurs, ils le firent rapidement connaître dans les tribus (dans la région de FortNapoléon il fut lu trois fois sur chaque marché), y voyant une garantie pour la propriété indigène avec d'autant plus de raison qu'ils n'avaient même pas à se justifier de ne pas appliquer la clause prévoyant l'établissement de la propriété individuelle puisque, officiellement, celle-ci était considérée comme se trouvant «déjà constituée, dans toutes les tribus kabyles, sur des bases aussi claires et aussi précises qu'en France » (rapport du général Allard au Sénat).

51 De plus, il apparut rapidement que l'exemple des colons n'avait pas sur les techniques des indigènes l'influence escomptée. On vit, au contraire, des colons adopter les méthodes traditionnelles et faire travailler leurs terres par des métayers indigènes. Certains, en outre, affectaient une attitude méprisante ou même brutale, sans parler de l'opposition permanente qu'ils manifestaient à l'égard de l'autorité militaire considérée, non parfois sans raisons, comme tyrannique. Et ainsi la lutte entre civils et militaires devint le trait dominant de la vie politique en Algérie sous le Second Empire.

L'aboutissement ce fut, après l'effondrement de l'Empire et la victoire des civils, le décret du 24 décembre 1870 supprimant le Bureau politique et les Bureaux arabes divisionnaires et subdivisionnaires, décret signé A. Crémieux, Fourichon, Glais-Bizoin et que Crémieux présentait le lendemain comme destiné «à rompre la hiérarchie des Bureaux arabes et la politique traditionnelle et antinationale que cette hiérarchie avait pour but de perpétuer. » Suivirent d'autre décrets dont le but était d'anéantir l'autorité militaire et notamment celle dévolue aux Bureaux arabes.

En réalité, ceux-ci ne disparurent que très progressivement. Si le Bureau arabe de FortNational est officiellement supprimé le 9 janvier 1872, on trouve encore, daté du 24 juillet 1874, un rapport d'inspection du capitaine Ravez «Chef du Bureau arabe de première classe de Fort-National ». C'est seulement en 1880 que toute la Grande Kabylie se trouva rattachée au territoire civil. Mais il restait 47 bureaux des affaires indigènes en Algérie et, en 1881, Maupassant visite certaines régions grâce à eux. En 1887, la division de Constantine employait toujours 40 à 45 officiers dans ses Bureaux arabes.

Il est vrai cependant qu'après 1870, l'autorité des Bureaux arabes est bien amoindrie et c'est sur leur action antérieure qu'il faut les juger sans pouvoir malheureusement, faute d'études régionales suffisantes, établir, s'il y eut des différences entre le pays berbère et le reste de l'Algérie. 
Il fut longtemps de règle de condamner sans appel le « régime du sabre ». Il apparaît aujourd'hui que le jugement dut être plus nuancé. Le bilan économique et social peut sembler bien mince, mais il faut l'estimer par rapport aux moyens dont disposaient les officiers et le comparer, pour une même durée de temps, à celui du régime civil. Au point de vue militaire et politique, ils ont ouvert la voie à la colonisation qui devait les combattre et ils ont créé rapidement les rouages d'une administration sans laquelle rien n'eût été possible. On oublie aussi leur apport à la vie scientifique et littéraire avec des noms comme Daumas, Hanoteau, Richard, Rinn, Margueritte, de Slane... ; il faudra des décennies aux civils pour assurer la relève, en accord, à ce moment, avec les officiers des Affaires Indigènes des Territoires du Sud, héritiers directs des anciens Bureaux arabes.

\section{BIBLIOGRAPHIE}

Nous n'indiquons que les études essentielles comportant elles-mêmes, le plus souvent, une importante bibliographie. Dans l'ordre chronologique :

YACONO X., Les Bureaux arabes et l'évolution des genres de vie indigènes dans l'ouest du Tell algérois (Dahra, Chélif, Ouarsenis, Sersou), Paris, Larose, 1953, 25 x 16,5 cm, 448 p. Une bibliographie de 185 titres.

GERMAIN R., La politique indigène de Bugeaud, Paris, Larose, 1955, 25 x 16,5 cm, 383 p. (pour les débuts des Bureaux arabes).

TURIN Y., Affrontements culturels dans l'Algérie coloniale, écoles, médecines, religion, Paris, Maspéro, $1971,22 \times 14$ cm, 434 p. Étude qui repose surtout sur les archives des Bureaux arabes de l'Algérois et du Constantinois.

SANGIOVANNI G., Le Bureau arabe de Fort-Napoléon (1857-1874). Travail d'étude et de recherche pour une maîtrise d'histoire, Université de Toulouse-Le Mirail, 1973, 271 pages dactylographiées. Une bibliographie de 60 titres.

cossu P.-P., l « Bureaux arabes » e il Bugeaud, Milan, Giufre, 1974, 24 x 17 cm, 135 p. Une bibliographie de 67 titres.

REY-GOLDZEIGUER A., Le royaume arabe. La politique algérienne de Napoléon III, 1861-1870, Alger, SNED, 1977, 814 p. Fait une large part à l'action des Bureaux arabes.

FRÉMEAUX J., Les Bureaux arabes dans la province d'Alger (1844-1856). Thèse de $3^{\mathrm{e}}$ cycle, Université de Toulouse-Le Mirail, 1976, texte dactylographié et photocopié, 512 pages en deux volumes. Une bibliographie de 146 titres.

A part :

PEYRONNET R, Livre d'or des officiers des Affaires indigènes, 1830-1930, Alger, 1930, 2 vol., $23 \times 16$ cm, 961 et 984 pages. 
INDEX

Mots-clés : Algérie, Histoire contemporaine, Politique, Sociologie 